\title{
New dilemmas in small-cell lung cancer TNM clinical staging
}

This article was published in the following Dove Press journal:

OncoTargets and Therapy

15 May 2013

Number of times this article has been viewed

Konstantinos Zarogoulidis'

Dimitrios Latsios'

Konstantinos Porpodis'

Paul Zarogoulidis ${ }^{1,2}$

Kaid Darwiche ${ }^{2}$

Nick Antoniou'

Wolfgang Hohenforst-

Schmidt ${ }^{3}$

Ellada Eleftheriadou'

Efimia Boutsikou'

Theodoros Kontakiotis'

'Pulmonary Department-Oncology Unit, "G Papanikolaou" General

Hospital, Aristotle University

of Thessaloniki, Thessaloniki, Greece;

${ }^{2}$ University Pulmonary DepartmentInterventional Unit, Ruhrlandklinik,

University, University of Duisburg-

Essen, Essen, Germany; ${ }^{3}$ II Medical

Clinic, Hospital of Coburg, University of Wuerzburg, Coburg, Germany
Correspondence: Paul Zarogoulidis Pulmonary Department-Oncology Unit, "G Papanikolaou" General Hospital, Aristotle University of Thessaloniki, Exohi I 100, Thessaloniki, Greece 57010 $\mathrm{Tel}+302310992433$

$\mathrm{Fax}+302310992433$

Email zarog@med.auth.gr
Background: Many patients with limited disease (LD) behave similarly to those with extensive disease (ED) from a prognostic point of view. On the other hand, a proportion of patients with ED small-cell lung cancer (SCLC) behave similarly to those with LD.

Patients and methods: In this retrospective study analysis, 764 patients with proven SCLC were included and managed with the same therapeutic protocols. Of these patients, $278(36.4 \%)$ had LD, while 486 (63.6\%) had ED.

Results: No statistically significant difference was observed for survival for IA and IB disease stages $(P=0.254)$ and between IIA and IIB stages $(P=0.256)$ according to the new tumor, node, metastasis (TNM) staging classification classification. In addition, no statistical significant difference was observed for survival between patients with (IIA + IIB) and IIIA $(P=0.951)$, (IIA + IIIA, $P=0.658$ ), and (IIB + IIIA, $P=0.573$ ) stages. Statistical significant difference was observed for survival among the LD SCLC patients with (IA + IB), (IIA + IIB + IIIA), and IIIB stages $(P<0.001)$. Similarly, statistical significance was observed for ED SCLC patients with (IIA + IIB + IIIA), IIIB, and IV stages $(P<0.001)$.

Conclusions: Although stratification of SCLC patients in LD and ED is generally satisfactory, the TNM staging system is recommended for more detailed prognostic information and treatment evaluation in these patients.

Keywords: small-cell lung cancer, staging, lung cancer

\section{Introduction}

Small-cell lung cancer (SCLC) represents approximately $15 \%$ of primary lung cancers overall. ${ }^{1}$ When considered independently from non-SCLC (NSCLC), SCLC is, by itself, the fifth leading cause of cancer mortality in the United States. ${ }^{2}$ The natural progress of the disease has been well documented with an average survival of less than 4 months, if left untreated. ${ }^{3}$ The introduction of chemotherapy to the treatment of SCLC has contributed to significant improvement in survival rates between the 1970s and 1990s. ${ }^{4}$

In lung cancer, the most important parameter is the pathologic determination of cell type. Given the pathology of SCLC, the anatomic extent of disease or stage provides prognostic information and treatment options. The first staging system for SCLC was introduced in the 1950s by the Veterans' Administration Lung Study Group (VALSG). ${ }^{5}$ This simple system defined patients as having either limited disease (LD) or extensive disease (ED) SCLC. LD SCLC was characterized by tumors confined to one hemithorax, which can be safely included in a single tolerable radiation portal. Local dissemination to ipsilateral and supraclavicular nodes but no extrathoracic metastases could be present. All other cases were classified as ED. 
In 1989, the International Association for the Study of Lung Cancer (IASLC) issued a consensus report ${ }^{6}$ that introduced changes to the VALSG staging system. This report suggested that local extension to regional lymph nodes, including contralateral mediastinal and contralateral supraclavicular, should be considered as LD. They also recommended that LD should be expanded to include ipsilateral pleural effusion, regardless of the cytology, in the absence of distant metastases.

The tumor, node, metastasis (TNM) staging systems of the American Joint Committee on Cancer Staging (AJCC) and the Union Internationale Contrele Cancer (UICC) are used less frequently in SCLC. ${ }^{7}$ The reason is that these two staging systems have historically relied on surgical confirmation for their accuracy, while the majority of patients with SCLC at the time of diagnosis have locally advanced or metastatic disease and, thus, are not eligible for surgical treatment. ${ }^{8}$ The 6th edition of the AJCC and UICC TNM staging system ${ }^{9}$ was revised to the current 7 th edition of the TNM lung cancer staging system, according to changes proposed by the IASLC. ${ }^{10,11}$ The majority of the changes were made in the $\mathrm{T}^{10}$ and $\mathrm{M}^{12}$ descriptors and the subsequent stage groupings. ${ }^{11}$

TNM staging in nonsurgical series has been reported rarely. ${ }^{13}$ Nevertheless, in 2007 the SCLC subcommittee of the IASLC Lung Cancer Staging Committee recommended that TNM staging be applied in SCLC. ${ }^{8}$

We herein report our survival analysis of clinical TNM staging for SCLC and assess the prognostic stratification based on the IASLC staging system. We focus on the clinical staging of SCLC because fewer than 5\% of patients are eligible for pathologic surgical staging at presentation. ${ }^{1}$

\section{Patients and methods Study design}

The database on which our analysis is based was created by the medical records of the Oncology Unit of the University Pulmonary Department, "G Papanikolaou” General Hospital, Thessaloniki, Greece.

We analyzed only patient cases that fulfilled the following criteria:

- Recruitment and follow-up of SCLC patients at a single institution, namely the Oncology Unit of the University Pulmonary Department at the "G Papanikolaou" General Hospital of Thessaloniki, Greece.

- Management of the disease along the same philosophy (chemotherapy, radiotherapy, dose and timing of radiotherapy, second-line therapy, palliative care).
- Availability of complete medical records (regarding history, laboratory findings, etc).

- Pathologic confirmation of the diagnosis by the same modality (tissue biopsy, cytologic material, etc).

- Performance status (PS) of the patients being either zero or one.

- From 779 subjects only 15 were excluded (all males) because of their inability in staging ( 7 with pleural effusion of unknown etiology and 8 because of limited data).

- Patients were deceased at time of analysis.

All the patients were managed with six to eight cycles of chemotherapy based on platinum analogs. Patients responding to chemotherapy were submitted to radiotherapy to the primary site (4.8 Gy over 5 weeks), and those with complete response also received prophylactic cranial irradiation. Partial responders received radiotherapy to the primary site (50 Gy), after chemotherapy completion. Complete responders also received prophylactic cranial radiation (29 Gy). Palliative radiotherapy and hematopoietic growth factors were administered as appropriate.

Patients with unknown or incomplete staging data were excluded. The stage classification was based on the following investigations: chest radiography; computed tomography of chest, abdomen, and brain; fiberoptic bronchoscopy; and isotope bone scans. In our retrospective study, we included the patients that had the same examinations for staging, since there were a greater number of patients that did not have positron-emission tomography (PET) scans, we excluded those that had PET scan. Additional tissue biopsies and cytology materials were obtained where appropriate.

Age, sex, smoking habit, and treatment options, including chemotherapy (first- and second-line), radiation therapy, and palliative care, were recorded. Dates of confirmed diagnosis, of presentation, of each medical intervention, and of death were also registered. $\mathrm{T}, \mathrm{N}$, and $\mathrm{M}$ categories were coded for each patient, according to both 6th edition AJCC/UICC ${ }^{9}$ and 7 th edition IASLC TNM staging systems. ${ }^{10,11}$ The descriptors and the derived disease stage were recorded separately for each classification system, as some patients were restaged according to the recommendations. ${ }^{10,11}$

\section{Analysis}

Survival in days was calculated for each patient, using an appropriate Microsoft Office Excel 2007 (Microsoft, Redmond, WA, USA) formula. Survival was measured from the date of confirmed diagnosis, either cytologically 
or histologically, and was estimated by the Kaplan-Meier method. Statistical significance was evaluated through log-rank, Breslow and Tarone-Ware tests. All statistical analyses were conducted using SPSS for Windows statistical software (release 13.0; IBM, Somers, NY, USA). Statistical significance was assumed for a two-tailed $P$ value less than 0.05 .

\section{Results}

\section{Patients and tumor demographics}

Incidence cases of SCLC were identified in the database of the Oncology Unit of the University Pulmonary Department, "G Papanikolaou” General Hospital, Thessaloniki, Greece. Pathologic confirmation of the diagnosis and adequate data for baseline LD or ED classification, TNM staging, and follow-up were required. A total of 764 patients met the inclusion criteria and comprised the final study population for this report. Date of confirmed diagnosis was between December 1988 and February 2011. The median age at diagnosis of the whole cohort of patients was 62.0 years (range 38-77 years; Table 1).

The 764 study patients were predominantly male $(\mathrm{n}=731 ; 95.7 \%)$ compared with female patients $(\mathrm{n}=33$; $4.3 \%)$. Of these patients, $536(70.2 \%)$ had a PS of 0 (according to World Health Organization [WHO] grading) and 228 $(29.8 \%)$ had a PS of 1 (Figure 1).

\section{Survival according to TNM stage}

All T, N, and M descriptors (T1a, T1b, T2a, T2b, T3, T4, N0, $\mathrm{N} 1, \mathrm{~N} 2, \mathrm{~N} 3, \mathrm{M} 1 \mathrm{a}$, and M1b) were recorded for each patient according to the latest IASLC revision. A small number of patients were included in each T, N, and M category, resulting from the relatively limited total number of 764 cases and the large number of derived patients groups (Table 2).

Survival analysis was performed in SCLC patients, based on the clinical 7 th edition of the IASLC TNM staging system
(Table 3). Statistical significance difference for survival was observed among disease stages $(P<0.001)$. Similar results were obtained based on the 6 th edition of the AJCC/ UICC TNM classification (data not shown). ${ }^{9}$ The median overall survival (OS) numbers of SCLC patients in our study are consistent with the results of the large Surveillance, Epidemiology and End Results (SEER) database of the National Cancer Institute. ${ }^{8}$ Similar findings were also reported by the IASLC database ${ }^{8}$ and the California Cancer Registry (CCR) database..$^{8,14}$

According to the analysis of our database, the median OS of stage IA (476 days) and stage IB (518 days) were reversed, whereas the median OS of stage IIA (359 days) and stage IIB (369 days) were essentially superimposed.

Differences in survival between IA and IB disease stages $(P=0.254)$ and between IIA and IIB stages $(P=0.256)$ were not statistically significant. These results provide plausible evidence to support the hypothesis that clinical stages IA and IB can be classified together, just like IIA and IIB stages. When performing the appropriate regrouping, patients were distributed in five stages (Table 3).

Statistical significant differences were observed in OS among the five disease stages $(P<0.001$; Table 3$)$. Nevertheless, the difference in OS between (IIA + IIB) and IIIA stages was not statistically significant $(P=0.951)$ and marginal in absolute values (369 vs 337 days). Moreover, prior to any regrouping, differences in OS between IIA and IIIA stages $(P=0.658)$ and between IIB and IIIA stages $(P=0.573)$ were not statistically significant. These results support the hypothesis that clinical stages IIA, IIB, and IIIA can be classified together (Table 3 ). Differences in survival between IA and IB disease stages $(P=0.254)$, between IIA and IIB stages $(P=0.256)$, between IIA and IIIA stages $(P=0.658)$, and between IIB and IIIA stages $(P=0.573)$. The difference in OS between (IIA + IIB) and IIIA stages was not statistically significant $(P=0.951)$ (Figure 1$)$.

Table I Distribution of patients, according to age at diagnosis and disease stage

\begin{tabular}{|c|c|c|c|c|c|}
\hline Age at diagnosis & Number of patients & Percentage of patients & Limited stage & Extensive stage & Total \\
\hline$<50$ years & 91 & $11.9 \%$ & & & \\
\hline $50-60$ years & 245 & $32.1 \%$ & & & \\
\hline $60-70$ years & 334 & $43.7 \%$ & & & \\
\hline$>70$ years & 94 & $12.3 \%$ & & & \\
\hline Stage IA + IB & & & 40 & 0 & 40 \\
\hline Stage IIA + IIB + IIIA & & & 180 & 31 & 211 \\
\hline Stage IIIB & & & 58 & 78 & 136 \\
\hline Stage IV & & & 0 & 377 & 377 \\
\hline Total & & & 278 & 486 & 764 \\
\hline
\end{tabular}


A

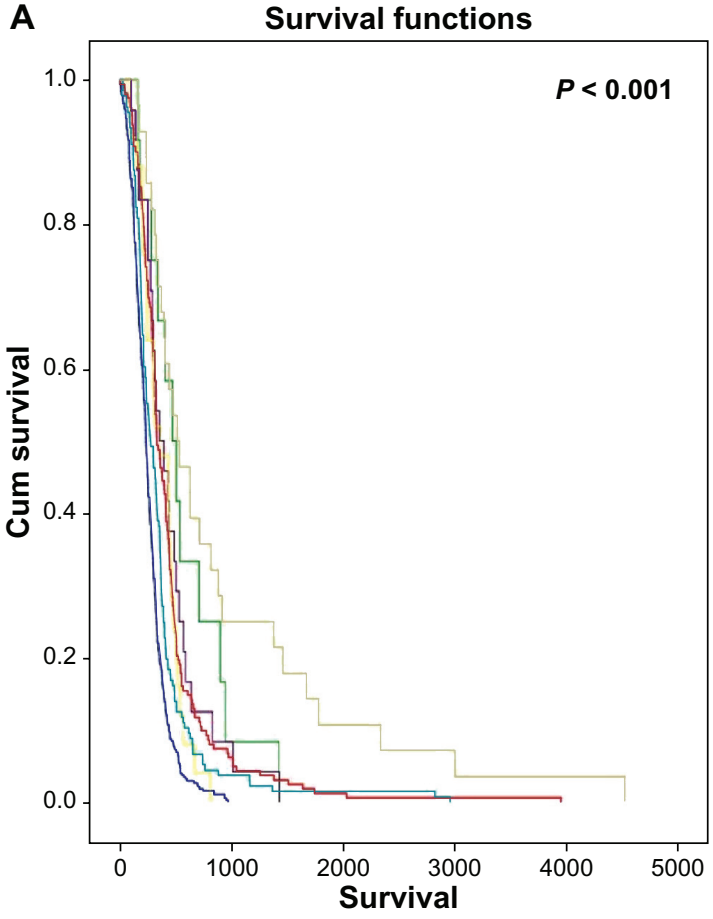

C

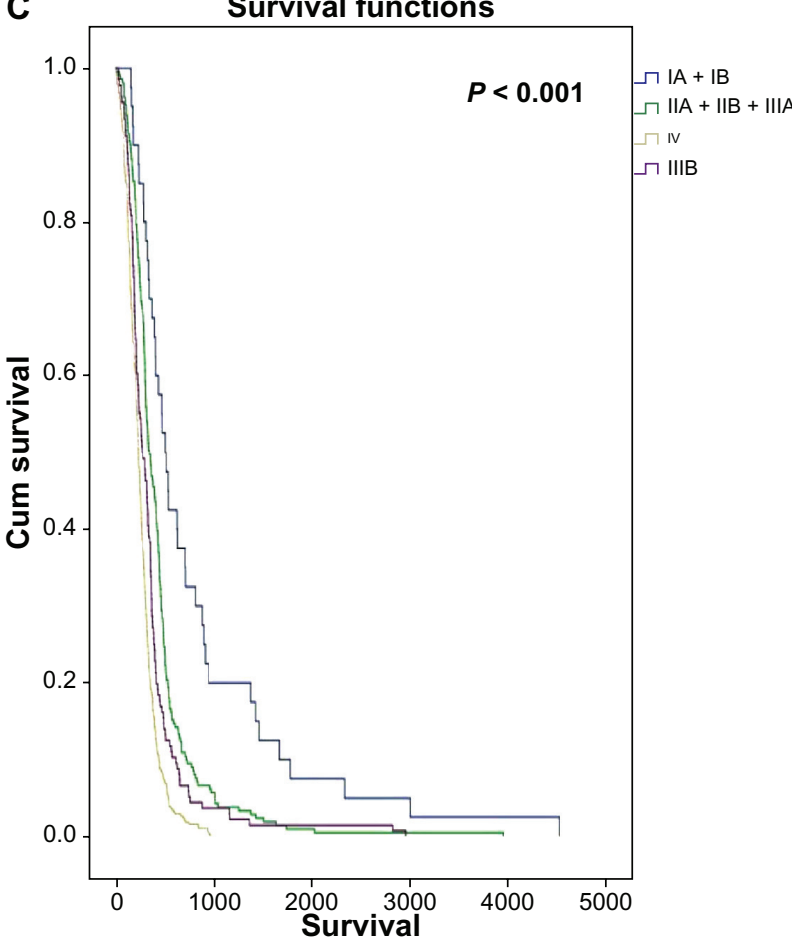

\section{B}

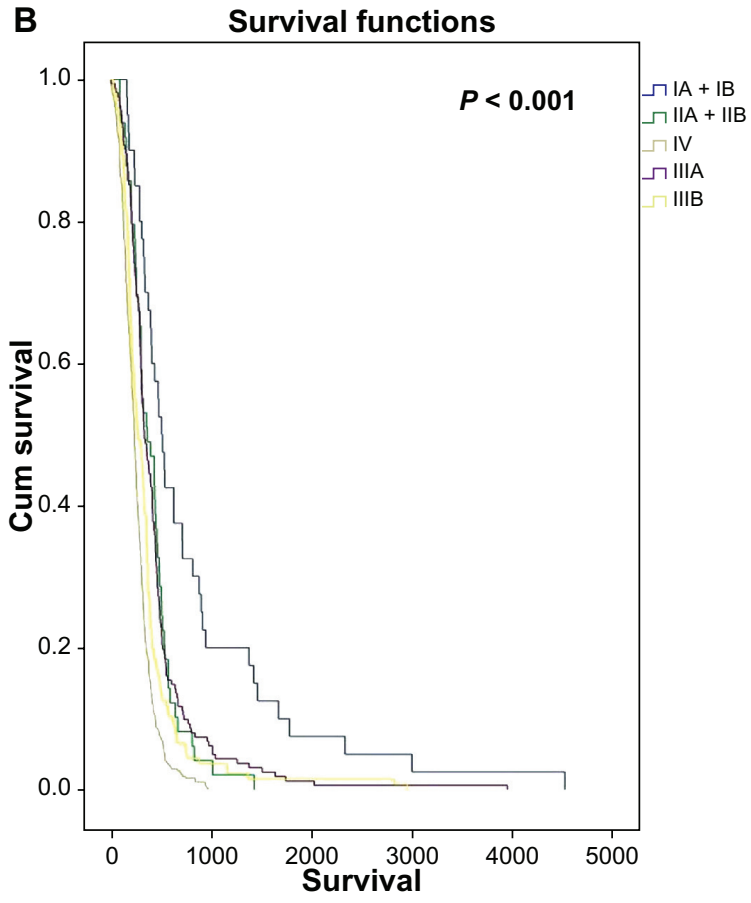

D

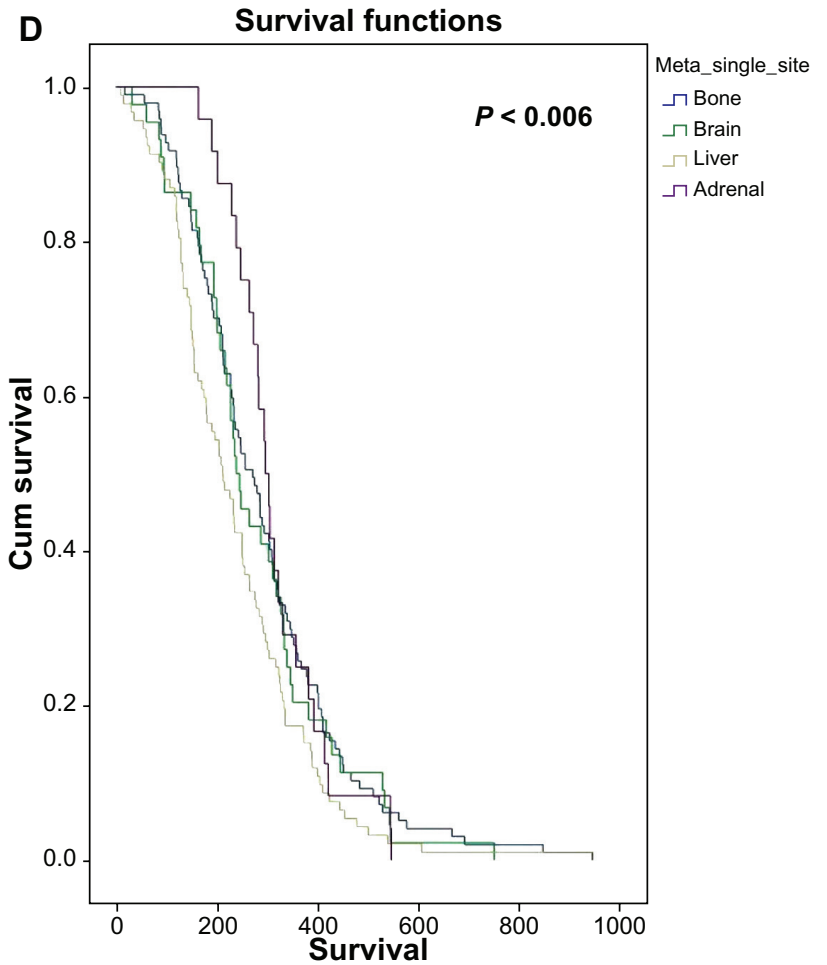

Figure I Survival analysis of proposed small cell lung cancer system (A) Survival analysis by disease stage, according to the 7th edition of the IASLC TNM staging system, (B) Survival analysis by disease stage, (C) Survival analysis by disease stage, (D) Survival analysis by single metastatic site. Abbreviations: IASLC, International Association for the Study of Lung Cancer; TNM, tumor, node, metastasis.

The latter regrouping introduces a newly proposed four-stage classification for SCLC patients. We found that there was statistically significant difference in OS among the four disease stages $(P<0.001)$. Survival between every one of the proposed four stages was different (Table 4) at a $<0.001$ level, except for the comparison between (IIA + IIB + IIIA) and IIIB stages $(P=0.004)$. This finding further contributes to the value of the proposed four-stage classification of SCLC patients (Figure 1). 
Table 2 Distribution of patients with respect to TNM descriptors, according to the 7th edition of the IASLC TNM classification system

\begin{tabular}{|c|c|c|c|}
\hline T category & $\mathbf{N}$ category & M category & Number of patients \\
\hline \multirow[t]{4}{*}{ la } & 0 & 0 & 44 \\
\hline & I & & I \\
\hline & 2 & & 10 \\
\hline & 3 & & 3 \\
\hline \multirow[t]{4}{*}{$\mathrm{Ib}$} & 0 & 0 & 8 \\
\hline & 1 & & 14 \\
\hline & 2 & & 16 \\
\hline & 3 & & 5 \\
\hline \multirow[t]{4}{*}{$2 a$} & 0 & 0 & 25 \\
\hline & I & & 5 \\
\hline & 2 & & 59 \\
\hline & 3 & & 18 \\
\hline \multirow[t]{4}{*}{$2 b$} & 0 & 0 & 3 \\
\hline & I & & 2 \\
\hline & 2 & & 13 \\
\hline & 3 & & 5 \\
\hline \multirow[t]{4}{*}{3} & 0 & 0 & 22 \\
\hline & 1 & & 3 \\
\hline & 2 & & 29 \\
\hline & 3 & & 16 \\
\hline \multirow[t]{4}{*}{4} & 0 & 0 & 23 \\
\hline & 1 & & 1 \\
\hline & 2 & & 59 \\
\hline & 3 & & 42 \\
\hline Any & Any & la & 19 \\
\hline Any & Any & $\mathrm{Ib}$ & 359 \\
\hline
\end{tabular}

Abbreviations: IASLC, International Association for the Study of Lung Cancer; TNM, tumor, node, metastasis.

\section{Comparison of the proposed four-stage SCLC patient classification with the stratification in either LD or ED}

In Table 1, we classified the 278 patients with LD according to the proposed four-stage classification. We also classified the 486 patients with ED according to the same four-stage classification. No patients with LD were classified as stage IV according to the TNM system, as expected. No patients with ED were classified as stage IA and IB.

We demonstrated that among 278 patients with LD, there are three subgroups with different behavior (Table 4). Patients with LD-SCLC and classified as IA or IB according to the TNM system had a median survival of 512 days. Patients with LD SCLC and classified as IIA or IIB or IIIA had a median survival of 360 days. Patients with LD SCLC and classified as IIIB had a median survival of 262 days. There was found a statistically significant difference in survival among these three subgroups of patients with $\operatorname{LD}(P<0.001)$. This finding implies that patients with LD are a heterogeneous group from
Table 3 Survival analysis by disease stage, according to the 7th edition of the IASLC TNM staging system

\begin{tabular}{llll}
\hline Disease stage & $\begin{array}{l}\text { Number } \\
\text { of patients }\end{array}$ & $\begin{array}{l}\text { Median survival } \\
\text { (days) }\end{array}$ & $\mathbf{9 5 \%} \mathbf{C l}$ \\
\hline Stage IA & 12 & 476 & $302.86-649.14$ \\
Stage IB & 28 & $5 I 8$ & $272.98-763.02$ \\
Stage IIA & 24 & 359 & $216.17-50 I .83$ \\
Stage IIB & 25 & 369 & $15 I .94-586.06$ \\
Stage IIIA & 162 & 337 & $286.4 I-387.59$ \\
Stage IIIB & 136 & 277 & $214.96-339.04$ \\
Stage IV & 377 & 236 & $220.39-25 I .6 I$ \\
Total & 764 & 286 & $270.47-30 I .53$ \\
Stage IA + IB & 40 & $5 I 2$ & $4 I 9.03-604.97$ \\
Stage IIA + IIB & 49 & 369 & $232.97-505.03$ \\
Stage IIIA & 162 & 337 & $286.4 I-387.59$ \\
Stage IIIB & 136 & 277 & $214.96-339.04$ \\
Stage IV & 377 & 236 & $220.39-25 I .6 I$ \\
Total & 764 & 286 & $270.47-30 I .53$ \\
Stage IA + IB & 40 & $5 I 2$ & $4 I 9.03-604.97$ \\
Stage IIA + IIB + IIIA & $2 I I$ & 348 & $296.63-399.37$ \\
Stage IIIB & 136 & 277 & $2 \mid 4.96-339.04$ \\
Stage IV & 377 & 236 & $220.39-25 I .6 I$ \\
Total & 764 & 286 & $270.47-30 I .53$ \\
\hline Abbreviation & &
\end{tabular}

Abbreviations: IASLC, International Association for the Study of Lung Cancer; Cl, confidence interval; TNM, tumor, node, metastasis.

a prognostic point of view. The different prognostic behavior of patients with LD can be expressed with the proposed fourstage classification based on TNM.

We performed a similar analysis in patients with ED (Table 4). Patients with ED SCLC classified as IIA or IIB or IIIA according to the TNM system had a median survival of 305 days. Patients with ED SCLC classified as IIIB had a median survival of 302 days. Patients with ED SCLC classified as IV had a median survival of 246 days. There was a statistically significant difference in survival among these three subgroups of patients with $\mathrm{ED}(P<0.001)$.

We also tried to compare the proposed four-stage classification of SCLC with the stratification in either LD or ED. We analyzed each of four stages separately and tested if there are statistically significant differences in survival between patients with LD and patients with ED, classified at the same stage. No such differences were found (data not shown). Therefore, the stratification of patients in LD and ED offers no additional prognostic information, when compared to the proposed four-stage classification of SCLC (Figure 1).

\section{Survival according to age at presentation}

Difference in survival among the predefined age groups was not statistically significant (Table 5; Figure 1). 
Table 4 Difference in OS between the four newly introduced disease stages and distribution by disease stage

\begin{tabular}{|c|c|c|c|c|c|c|c|c|}
\hline \multirow[t]{2}{*}{ Disease stage } & \multirow[t]{2}{*}{ Stage IA + IB } & \multirow[t]{2}{*}{ Stage IIA + IIB + IIIA } & \multirow[t]{2}{*}{ Stage IIIB } & \multirow[t]{2}{*}{ Stage IV } & \multicolumn{2}{|c|}{ Limited disease } & \multicolumn{2}{|c|}{ Extensive disease } \\
\hline & & & & & MS & $95 \% \mathrm{Cl}$ & MS & $95 \% \mathrm{Cl}$ \\
\hline Stage IA + IB & NA & $P<0.001$ & $P<0.001$ & $P<0.001$ & 512 & $419.03-604.97$ & NA & \\
\hline Stage IIA + IIB + IIIA & $P<0.001$ & NA & $P=0.004$ & $P<0.001$ & 360 & $304.49-4|5.5|$ & 305 & $239.56-370.44$ \\
\hline Stage IIIB & $P<0.00 I$ & $P=0.004$ & NA & $P<0.001$ & 262 & $187.36-336.63$ & 302 & $205.71-398.29$ \\
\hline \multirow[t]{2}{*}{ Stage IV } & $P<0.001$ & $P<0.00 I$ & $P<0.001$ & NA & 359 & $315.82-402.18$ & 246 & $231.07-260.93$ \\
\hline & & & & $P<0.001$ & & & $P<0.001$ & \\
\hline
\end{tabular}

Abbreviations: $\mathrm{Cl}$, confidence interval; MS, median survival; NA, not available; OS, overall survival.

\section{Survival according to metastatic site}

There was a statistically significant difference in survival among patients with different number of metastatic sites at diagnosis $(P<0.001$; Table 5$)$. An increasing number of metastatic sites was related with progressively worse survival.

We specifically examined the cases with a single metastatic site. We found that the site of the metastasis is associated with survival $(P=0.006)$. Single liver metastasis, found in 92 patients, is associated with shorter survival (212 days), which is statistically significant when compared with single bone $(P=0.010)$ and single adrenal metastasis $(P=0.003)$ (Table 5). Difference in survival between patients with single liver and single brain metastasis was insignificant $(P=0.092)$. Single brain metastasis, detected in 44 patients, is also associated with shorter survival (239 days), which is not statistically significant when compared with single bone $(P=0.691)$, single liver $(P=0.092)$ and single adrenal metastasis $(P=0.478)$. However, due to limited number of patients, it is difficult to determine the prognostic significance of single brain metastasis in SCLC patients (Figure 1).

\section{Discussion}

Approximately $15 \%$ of all primary lung cancers are small-cell carcinomas. However, in large surgical series, patients with SCLC represent less than $5 \%$ of cases, which is attributed to the early dissemination at time of diagnosis. ${ }^{1}$ The use of TNM descriptors has been the cornerstone of the NSCLC staging system since 1973. Nevertheless, most clinicians have not used TNM descriptors and prefer a two-stage classification in either LD or ED. ${ }^{5,6}$

The traditional opinion has been that more precise staging of SCLC, as a result of TNM system application, does not offer extra guidance to treatment selection. Nevertheless, a more precise definition of nodal involvement may be of particular importance for effective radiation therapy. In the past, the common radiotherapy ports for chest irradiation were large, where only moderate dose delivery was possible. This limitation is possibly reflected on high rates of treatment failure at the primary site, reported in most studies evaluating thoracic radiotherapy. According to the 1989 IASLC staging system for SCLC, involvement of ipsilateral and contralateral supraclavicular nodes and of ipsilateral and contralateral hilar or mediastinal nodes is classified as LD. In the modern era of conformal techniques and increasing radiation dose, accurate radiotherapy planning is vital. Therefore, reduction of the radiotherapy field size is possible and advisable, based on more precise nodal involvement determination. ${ }^{8}$

Moreover, the SCLC subcommittee of the IASLC Lung Cancer Staging Project recommends that TNM staging stratification in SCLC should be incorporated into all trials in LD SCLC, particularly those addressing thoracic and prophylactic cranial irradiation issues and those including a surgical treatment arm. The small survival differences expected in these trials could be affected significantly by patients subgrouping. ${ }^{8}$ Stratification by stages I-III should be

Table 5 Survival analysis by age at diagnosis, by number of metastatic sites

\begin{tabular}{llll}
\hline $\begin{array}{l}\text { Age at } \\
\text { diagnosis }\end{array}$ & $\begin{array}{l}\text { Number } \\
\text { of patients }\end{array}$ & $\begin{array}{l}\text { Median survival } \\
\text { (days) }\end{array}$ & $95 \% \mathbf{C l}$ \\
\hline$<50$ years & 91 & $29 I$ & $260.4 I-321.59$ \\
$50-60$ years & 245 & 288 & $256.09-319.91$ \\
$60-70$ years & 334 & 282 & $256.93-307.07$ \\
$>70$ years & 94 & 273 & $228.95-317.05$ \\
Number of different metastatic sites & 334 & $307.87-360.13$ \\
0 & 395 & $25 I$ & $228.99-273.01$ \\
I & 270 & 180 & $128.52-231.48$ \\
2 & 88 & 152 & $67.57-236.43$ \\
3 & 11 & 286 & $270.47-301.53$ \\
Total & 764 & & $228.13-315.87$ \\
Metastatic sites & 97 & 272 & $198.91-279.09$ \\
Bone & 44 & 239 & $171.58-252.42$ \\
Brain & 92 & 212 & $270.59-323.40$ \\
Liver & 24 & 297 & $225.72-272.27$ \\
Adrenal & 257 & 249 &
\end{tabular}

Abbreviation: $\mathrm{Cl}$, confidence interval. 
performed in all clinical trials for early-stage SCLC whether treatment is surgical or not.

Nevertheless, the value of routinely applying the TNM system to all SCLC patients remains controversial. Exact TNM stage is unlikely to alter treatment decisions for the vast majority of patients with $\mathrm{LD}$, because the best treatment option is combined modality therapy to patients with good performance status in most subsets of stages I, II, and III. ${ }^{8}$

The 7th edition of the IASLC TNM staging system was applied to the large SEER database of the National Cancer Institute. ${ }^{8}$ The median survival of stages IIA, IIB, IIIA, and IIIB was essentially the same, ranging from 11-15 months. There was also a concordance in median survival between stages IA and IB.

Similar findings were reported by the IASLC database, with the exception of the extremely small subgroup of eight patients with stage IIA. ${ }^{8}$ Pairwise comparisons between adjacent stage groupings revealed significant differences, although the hazard ratio for IIA versus IB was the reverse of the expected. However, the absolute median survival differences between stage IA and IB and among stages IIB, IIIA, and IIIB were relatively small.

A validation study of the 7 th edition of the IASLC TNM staging system, using the CCR database ${ }^{14}$ revealed statistically not significant survival differences between stages IIA and IB $(P=0.9070)$, IIB and IIA $(P=0.1401)$, and between IIIA and IIB $(P=0.3238)$.

In the latter study, based on the CCR database,,${ }^{14}$ pairwise comparisons between $\mathrm{T}$ descriptors did not present statistical significance between T2 versus T3. In the IASLC report, ${ }^{8}$ patients with clinical stages T2, T3, and T4 had similar median survival $(15,13$, and 12 months, respectively), although statistically different. In both studies, the difference between $\mathrm{T} 1$ and $\mathrm{T} 2$ was the largest and represented the primary separation for clinical $\mathrm{T}$ category. Likewise, the largest modern series of surgically resected SCLC cases did not present statistically significant differences in median survival between $\mathrm{T} 2$ and $\mathrm{T} 3$ and between $\mathrm{T} 3$ and T4 groups. ${ }^{1}$

Pairwise comparisons of the hazard ratio between sequential $\mathrm{N}$ descriptors in the CCR database report, ${ }^{14}$ also revealed nonsignificance between $\mathrm{N} 1$ versus $\mathrm{N} 0$, which was similar to the findings reported by IASLC ${ }^{8}$ Moreover, both in the IASLC report and in the CCR database analysis, the difference in median survival between N2 and N3 stages, although statistically significant, was marginal in absolute values (14 versus 12 months, according to the IASLC report, and 10 versus 9 months, according to the CCR database).
Results of the CCR database showed that nodal status failed to differentiate survival of T4 nonmetastatic (M0) patients. In both studies, the difference between N1 and N2 represented the primary separation for clinical $\mathrm{N}$ category. Nodal status was also proven inferior to the $\mathrm{T}$ descriptor at prognosticating early-stage SCLC patients. Moreover, clinical nodal staging was found to be in poor concordance with the pathologic $\mathrm{N}$ category in patients with SCLC who underwent surgical resection. ${ }^{1}$

As demonstrated by the aforementioned results from large-scale studies in SCLC, the median survival differences among $\mathrm{T}$ and $\mathrm{N}$ descriptors and TNM stages are not widely separated. In particular, statistically significant differences in OS were demonstrated which were not striking in absolute terms and of doubtful clinical importance. According to the authors of this study, these data also provide plausible evidence to support the hypothesis that the clinical staging of SCLC, even according to the latest recommendations proposed by IASLC and its well-established advantages, has potential vulnerabilities.

Large-scale series on SCLC staging included patients managed with different therapeutic philosophy and in various treatment centers. ${ }^{1,8,14}$ Therefore, there was no uniform protocol on how treatment (surgery, radiation, or chemotherapy) was given in these retrospective studies. This limitation was partly eliminated by the inclusion of many independent prognostic factors into the Cox proportional regression analysis. In most studies, survival was recorded to the nearest month and a minority of patients were still alive at the end of the study, ${ }^{1,8,14}$ These parameters limit the accuracy of the survival analysis.

The goal of this study was to perform a retrospective survival analysis of patients with SCLC managed with the same therapeutic protocol at a single pulmonary oncology department.

Patients managed in other oncology centers were not included in our study, because of different therapeutic philosophy, concerning radiotherapy, second-line therapy, and palliative treatment. The different treatment philosophies would possibly reflect conflicting survival results.

In addition, all patients included in our study were deceased at time of analysis. Survival in days was calculated for each patient, starting from the date of confirmed diagnosis.

Therefore, the IASLC-proposed definitions of T, N, and $\mathrm{M}$ descriptors and TNM stage groupings for SCLC were applied to the database of our patients. Due to relatively limited total number of 764 cases and the consequent small number of patients included in each T, N, and M category, 
the prognostic significances of the $\mathrm{T}, \mathrm{N}$, and $\mathrm{M}$ descriptors were not examined individually.

All 764 patients in our dataset were classified according to both the 6th edition AJCC/UICC and the 7th edition IASLC TNM staging systems. The results were similar as a result of the limited number of patients needing reclassification between these two different stage groupings.

We reported our survival analysis of clinical TNM staging for SCLC and assessed the prognostic stratification based on the IASLC staging system. Our findings on survival characteristics are compatible with the results reported by the SEER database of the National Cancer Institute, the IASLC database, and the CCR database.

According to the analysis of our database, the median OS of stage IA (476 days/15.9 months) and stage IB (518 days/17.3 months) were reversed. This result could be attributed to the limited number of patients classified in these clinical stages (12 and 28 patients, respectively). A similar finding was reported by the IASLC database (31 and 35 months, respectively). ${ }^{8}$

Our results also showed a near superimposition of OS between stage IIA (359 days/12 months) and stage IIB (369 days/12.3 months). In the SEER database along with the CCR database, stage IIA SCLC patients (15 and 19 months, respectively) had slightly superior survival compared to IIB cases (12 and 15 months, respectively).

Moreover, we found that OS of stage IIA and IIB patients (359 days/12 months and 369 days/12 months, respectively) was slightly superior, but statistically insignificant compared to the IIIA patients (337 days/11.2 months). The median OS of stage IIA, IIB, and IIIA patients was found to be 15, 12, and 13 months in the SEER database and 19, 15, and 14 months in the CCR database, respectively.

These results support the hypothesis that clinical stages IA and IB can be classified together, as well as the IIA, IIB, and IIIA stages. The latter regrouping introduces a new four-stage classification for SCLC patients. Our hypothesis is also supported by the aforementioned results from large-scale studies in SCLC, which revealed marginal median survival differences among $\mathrm{T}$ and $\mathrm{N}$ descriptors and TNM stages. Moreover, our proposed four-stage classification of SCLC based on TNM system was proved to be superior to stratification in either LD or ED at prognosticating SCLC patients.

Perhaps some factors other than current TNM descriptors need to be further investigated towards SCLC-staging optimization. There is increasing evidence that the expression levels of potentially predictive biomarkers of chemotherapeutic efficacy have clinical utility in SCLC. ${ }^{4}$

This study is retrospective in nature and thus has the limitations of population-based studies. There was no centralized review of pathologic specimens as the definition of SCLC has evolved throughout the years. ${ }^{15}$ However, we have excluded patients with combined SCLC histology, according to the new WHO lung tumor classification. ${ }^{16}$ Secondly, there is an emphatic male predominance in population of our study. A possible explanation is that smoking prevalence in Greece is greater in men than in women. The smoking habit, the leading risk factor for SCLC, is similar between young males and females in Greece, but this is not the case with the older clusters of population. Even nowadays, women in rural areas smoke less, possibly owing to the existing traditional culture that regards female smoking a taboo. ${ }^{17}$ Moreover, the staging strategy for SCLC proposed by the current study is based on clinical evaluation of the extent of disease. The true extent of the SCLC disease is not always evident from the staging investigations (hematology, biochemistry, bronchoscopy, computed tomography and isotope scans) used in our study, including magnetic resonance imaging. Clinical staging often underestimates the extent of SCLC, as imaged by conventional radiology. The discrepancy between clinical and pathologic staging in SCLC was demonstrated in several studies. ${ }^{1,18,19}$ As SCLC is a highly metabolic tumor that avidly takes up fluorodeoxyglucose, PET scanning could be an attractive modality for staging. In small prospective studies, PET scans have correctly upstaged patients..$^{20,21}$ The main limitations of PET use are its cost, its limited availability, and the lack of expertise in performing the examination, so most oncologists do not use this method in everyday practice for SCLC staging. We didn't use PET scans in this retrospective study. ${ }^{22}$ There is also increasing evidence that the expression levels of potentially predictive biomarkers of chemotherapeutic efficacy have clinical utility in SCLC. In particular, ribonucleotide reductase 1 and topoisomerase $2 \alpha$ may provide an alternative to the onesize-fits-all chemotherapy in SCLC. ${ }^{4}$

In conclusion, we can state that the new proposed fourstage classification in SCLC is more accurate in predicting survival compared with the traditional used LD and ED staging. On the other hand, the proposed new IASLC staging is inaccurate for SCLC because in most of the 22 subgroups there is no significant OS difference. More prospective studies could be useful in order to validate these retrospective findings. 


\section{Disclosure}

The authors declare no conflicts of interest in this work.

\section{References}

1. Vallieres E, Shepherd FA, Crowley J, et al. The IASLC Lung Cancer Staging Project: proposals regarding the relevance of TNM in the pathologic staging of small cell lung cancer in the forthcoming (seventh) edition of the TNM classification for lung cancer. JThorac Oncol. 2009; 4(9):1049-1059.

2. Argiris A, Murren JR. Staging and clinical prognostic factors for smallcell lung cancer. Cancer J. 2001;7(5):437-447.

3. Hann CL, Rudin CM. Management of small-cell lung cancer: incremental changes but hope for the future. Oncology (Williston Park). 2008;22(13):1486-1492.

4. Chiappori AA, Zheng Z, Chen T, et al. Features of potentially predictive biomarkers of chemotherapeutic efficacy in small cell lung cancer. J Thorac Oncol. 2010;5(4):484-490.

5. Zelen M. Keynote address on biostatistics and data retrieval. Cancer Chemother Rep 3. 1973;4(2):31-42.

6. Stahel RA, Ginsberg RJ, Havemann K, Hirsch FR, Ihde DC, Jassem J. Staging and prognostic factors in small cell lung cancer: a consensus report. Lung Cancer. 1989;5:119-126.

7. Detterbeck FC, Boffa DJ, Tanoue LT. The new lung cancer staging system. Chest. 2009;136(1):260-271.

8. Shepherd FA, Crowley J, Van Houtte P, et al. The International Association for the Study of Lung Cancer lung cancer staging project: proposals regarding the clinical staging of small cell lung cancer in the forthcoming (seventh) edition of the tumor, node, metastasis classification for lung cancer. J Thorac Oncol. 2007;2(12): 1067-1077.

9. Sobin L, Wittekind C. UICC International Union Against Cancer: TNM Classification of Malignant Tumours, 6th ed. New York: WileyBlackwell; 2002.

10. Rami-Porta R, Ball D, Crowley J, et al. The IASLC Lung Cancer Staging Project: proposals for the revision of the T descriptors in the forthcoming (seventh) edition of the TNM classification for lung cancer. $J$ Thorac Oncol. 2007;2(7):593-602.
11. Goldstraw P, Crowley J, Chansky K, et al. The IASLC Lung Cancer Staging Project: proposals for the revision of the TNM stage groupings in the forthcoming (seventh) edition of the TNM Classification of malignant tumours. J Thorac Oncol. 2007;2(8):706-714.

12. Postmus PE, Brambilla E, Chansky K, et al. The IASLC Lung Cancer Staging Project: proposals for revision of the $\mathrm{M}$ descriptors in the forthcoming (seventh) edition of the TNM classification of lung cancer. J Thorac Oncol. 2007;2(8):686-693.

13. Shepherd FA, Ginsberg RJ, Haddad R, et al. Importance of clinical staging in limited small-cell lung cancer: a valuable system to separate prognostic subgroups. The University of Toronto Lung Oncology Group. J Clin Oncol. 1993;11(8):1592-1597.

14. Ignatius Ou SH, Zell JA. The applicability of the proposed IASLC staging revisions to small cell lung cancer (SCLC) with comparison to the current UICC 6th TNM Edition. J Thorac Oncol. 2009;4(3): 300-310.

15. Ettinger DS, Aisner J. Changing face of small-cell lung cancer: real and artifact. J Clin Oncol. 2006;24(28):4526-4527.

16. Brambilla E, Travis WD, Colby TV, Corrin B, Shimosato Y. The new World Health Organization classification of lung tumours. Eur Respir J. 2001;18(6):1059-1068.

17. Vardavas CI, Kafatos AG. Smoking policy and prevalence in Greece: an overview. Eur J Public Health. 2007;17(2):211-213.

18. Sakai M, Ishikawa S, Yamamoto T, et al. Preoperative TNM evaluation of peripheral clinical stage I small cell lung cancer treated by initial lobectomy with adjuvant chemotherapy. Interact Cardiovasc Thorac Surg. 2005;4(2):118-122.

19. Inoue M, Nakagawa K, Fujiwara K, Fukuhara K, Yasumitsu T. Results of preoperative mediastinoscopy for small cell lung cancer. Ann Thorac Surg. 2000;70(5):1620-1623.

20. Bradley JD, Dehdashti F, Mintun MA, Govindan R, Trinkaus K, Siegel BA. Positron emission tomography in limited-stage small-cell lung cancer: a prospective study. J Clin Oncol. 2004;22(16):3248-3254.

21. Chin R Jr, McCain TW, Miller AA, et al. Whole body FDG-PET for the evaluation and staging of small cell lung cancer: a preliminary study. Lung Cancer. 2002;37(1):1-6.

22. Irshad A, Ravenel JG. Imaging of small-cell lung cancer. Curr Probl Diagn Radiol. 2004;33(5):200-211.
OncoTargets and Therapy

\section{Publish your work in this journal}

OncoTargets and Therapy is an international, peer-reviewed, open access journal focusing on the pathological basis of all cancers, potential targets for therapy and treatment protocols employed to improve the management of cancer patients. The journal also focuses on the impact of management programs and new therapeutic agents and protocols on

\section{Dovepress}

patient perspectives such as quality of life, adherence and satisfaction. The manuscript management system is completely online and includes a very quick and fair peer-review system, which is all easy to use. Visit http://www.dovepress.com/testimonials.php to read real quotes from published authors. 